\title{
STRESS COMPONENTS FOR THEMOELASTIC INFINITE \\ PLATE WITH A CURVILINEAR HOLE BY USING COMPLEX VARIABLES METHOD.
}

A. A. EL-BARY

(Received 28 February, 2004; Revision accepted 22 April, 2004).

\section{ABSTRACT}

Different methods are established to solve the mixed problem in the theory of elasticity and thermoelasticity. Thermoelasticity represents a generalization of the theory of elasticity, so the equations characterizing the particular material and temperature dependence include an additional relation connecting the heat flux in the body with local temperature gradient.

In this paper we shall use the Cauchy Complex Variables method to solve the integro-differential equation to obtain the components of stress, in the presence of uniform flow of heat in negative direction of $y$-axis for an infinite plate with a curvilinear hole.

KEYWORDS: Theory of thermoelasticity, stress components, infinite plate, curvilinear hole, complex variable method .

A very powerful method to solve the integro-differential equation in the thermoelastic plates is discussed by (Parkus, 1976). Also, many authors such, Abdou $(1994,2001)$, El-Bary $(2000,2001,2003)$, Aleksandrov \& Kovalenkov (1986),

El-Sirafy ( 1977) and Muskhelishvili ( 1949) discussed the solution of elastic infinite plate with a curvilinear hole due to different types of solution in the case of absence of heat.

El-Bary (2000), England (1971), Koya \& Erdogan (1997) and Popv ( 1982) considered some important problems in integral and integro-differential equations and gave the solution. Then we use the solution of first and second fundamental problems of infinite elastic plate and the solution of integro-differential with Cauchy kernel to solve our problem in the case of thermoelastic plate with a curvilinear hole having two poles.

\section{Basic equations:}

Aleksandrov, Kovalenkov, England, Muskhelishvili, Parkus and Popv format and found the Goursat's function for the first and second fundamental problems and they found the two analytic functions $\Phi(z)$ and $\psi(z)$ which satisfy the boundary condition:

$$
k \phi(t)-t \overline{\phi^{\prime}(t)}-\overline{\psi(t)}=f(t)
$$

The authors solve these problems according to simple type of transformations. In [3], authors generalize the transformations and solve the same problems and some applied examples. In this paper we discuss thermoelastic infinite plate with a curvilinear hole having two poles, so our transformation is:

$$
Z=c u(z)=c \frac{\xi+m_{1} \xi^{-1}+m_{2} \xi^{-2}}{\left(1-n_{1} \xi^{-1}\right)\left(1-n_{2} \xi^{-1}\right)}, \quad c>0, \quad n_{1} \neq n_{2}
$$


and as in [11], we use Cauchy integral method to obtain $\Phi(z)$ and $\psi(z)$. From [14], q, the rate of heat flow per unit area is proportional to the temperature gradient in the direction of the normal $n$ to the element and $q=-k \frac{\partial \theta}{\partial n}$, where $k$ is the thermal conductivity of the material, $\theta$ is the temperature increase and the minus sign is due to the fact that heat flows in the direction of decreasing temperature. For the stationary case (time independent) we have: $\nabla^{2} \theta=0$, i.e. $\theta(x, y)$ satisfies Laplace equation.

\section{Mapping Function:}

We use the transformation:

$Z=\mathrm{cw}(\mathrm{z})=\mathrm{c} \frac{\xi+m_{1} \xi^{-1}+m_{2} \xi^{-2}}{\left(1-\mathrm{n}_{1} \xi^{-1}\right)\left(1-\mathrm{n}_{2} \xi^{-1}\right)}, \quad \mathrm{c}>0, \quad \mathrm{n}_{1} \neq \mathrm{n}_{2}$

We can write:

$\frac{Z}{\overline{Z^{\prime}}}=\alpha(\xi)+\overline{\beta(\xi)}$

where $\beta(\xi)$ is a regular part and $\alpha(\xi)$ is a singular part.

Also, we can write $\alpha(\xi)$ in the form:

$\alpha(\xi)=\frac{h_{1}}{\xi-n_{1}}+\frac{h_{2}}{\xi-n_{2}}$

where $h_{1}, h_{2}$ are real constants and can be calculated as:

$n_{1}=\frac{\left(n_{1}^{3}-m_{1} n_{1}+m_{2}\right)\left(1-n_{1}^{2}\right)^{2}\left(1-n_{1} n_{2}\right)^{2}}{\left(n_{1}-n_{2}\right)\left[\left(1-n_{1}^{2}\right)\left(1-n_{1} n_{2}\right)\left(3+m_{1} n_{1}^{2}\right)-\left(2-n_{1}^{2}-n_{1} n_{2}\right)\left(1+m_{1} n_{1}^{2}+m_{2} n_{1}^{3}\right)\right]}$

to calculate $h_{2}$, replace $n_{1}$ by $n_{2}$ and $n_{2}$ by $n_{1}$ in $h_{1}$

$h_{2}=\frac{\left(n_{2}^{3}-m_{1} n_{2}+m_{2}\right)\left(1-n_{2}^{2}\right)^{2}\left(1-n_{1} n_{2}\right)^{2}}{\left.\left(n_{2}-n_{1}\right)\left(1-n_{2}^{2}\right)\left(1-n_{1} n_{2}\right)\left(3+m_{1} n_{2}^{2}\right)-\left(2-n_{2}^{2}-n_{1} n_{2}\right)\left(1+m_{1} n_{2}^{2}+m_{2} n_{2}^{3}\right)\right]}$

Using (3.2) in (2.1), we get:

$\mathrm{k} \phi(\sigma)-\frac{\mathrm{w}(\sigma)}{\overline{\mathrm{w}^{\prime}(\sigma)}} \overline{\phi^{\prime}(\sigma)}-\overline{\psi(\sigma)}=\mathrm{f}(\sigma)$

Again, use (3.2) in (3.7)

$k \phi(\sigma)-\alpha(\sigma) \overline{\phi^{\prime}(\sigma)}-\overline{\psi_{0}(\sigma)}=\mathrm{f}_{\cdot}(\sigma)$

where 
$\psi \cdot(\xi)=\psi(\xi)+\beta(\xi) \phi^{\prime}(\xi)$,

f. $(\xi)=F(\xi)-c k \Gamma \xi+c \bar{\Gamma}^{*} \xi^{-1}+N(\xi)[\alpha(\xi)+\bar{\beta}(\xi)]$

and

$\mathrm{N}(\xi)=\mathrm{c} \vec{\Gamma}-\frac{X+\mathrm{i} Y}{2 \pi(1+\chi)}$

$F(\xi)=f(Z)=f(\operatorname{cw}(\xi))$

Also, as in [3], we can write

$\mathrm{N}\left(\mathrm{n}_{\mathrm{j}}\right)=\mathrm{c} \bar{\Gamma}-\frac{\mathrm{X}-\mathrm{iY}}{2 \pi(1+\chi)} \mathrm{n}_{\mathrm{j}}, \quad(\mathrm{j}=1,2)$

Multiplying (3.8) by $\frac{1}{2 \pi i(\sigma-\xi)}$ then integrating on $\gamma$ with respect to do, we get:

$\mathrm{K} \phi(\xi)+\frac{1}{2 \pi i} \int_{\gamma_{j}} \frac{\alpha(\sigma) \overline{\phi^{\prime}(\sigma)}}{\sigma-\xi} \mathrm{d} \sigma=\frac{\mathrm{c} \overline{\Gamma^{*}}}{\xi}-\mathrm{A}(\xi)+\sum_{\mathrm{j}=1}^{2} \frac{\mathrm{h}_{\mathrm{j}} \mathrm{N}_{\mathrm{j}}}{\xi-\mathrm{n}_{\mathrm{j}}}$

where $A(\xi)=\frac{1}{2 \pi i} \int_{\gamma_{j}} \frac{F(\sigma)}{\sigma-\xi} d \sigma$

Using (3.4) in (3.9), we have:

$\frac{1}{2 \pi \mathrm{i}} \int_{\gamma}^{\alpha(\sigma) \overline{\phi_{\mathrm{j}}^{\prime}}(\sigma)} \frac{\mathrm{\sigma}-\xi}{\sigma-\xi} \mathrm{d}=\mathrm{\sum} \sum_{\mathrm{j}=1}^{2} \frac{\mathrm{b}_{\mathrm{j}} \mathrm{h}_{\mathrm{j}}}{\mathrm{n}_{\mathrm{j}}-\xi}$

where $b_{j}(j=1,2)$ are complex constants to be determined

$-K \phi(\xi)=A(\xi)-\frac{c \overline{\Gamma^{*}}}{\xi}+\sum_{j=1}^{2} \frac{h_{j}}{n_{j}-\xi}\left(c b_{j}+N_{j}\right)$

Inserting $\overline{\phi^{\prime}(\sigma)}$ from $(3.10)$ to $(3.9)$, we get:

$\mathrm{Kcb}_{\mathrm{j}}+\mathrm{h}_{\mathrm{j}} \mathrm{V}_{\mathrm{j}}\left(\overline{c b_{j}}+\mathrm{N}_{\mathrm{j}}\right)+\mathrm{cn}_{\mathrm{j}}^{2} \Gamma^{*}+\overline{\mathrm{A}^{\prime}\left(\mathrm{n}_{\mathrm{j}}^{-1}\right)}=0, \quad \mathrm{j}=1,2$

where

$V_{j}=\frac{n_{j}^{2}}{1-n_{j}^{2}}$ 
$c b_{j}=\frac{K_{j}-h_{j} N_{j} E_{j}}{K^{2}-h_{j}^{2} V_{j}^{2}}, \quad(j=1,2)$, and

$-E_{j}=A^{\prime}\left(n_{j}^{-1}\right)+\operatorname{cn}_{j}^{2} \Gamma^{*}+h_{j} V_{j} N_{j}$

Then, we can find $\psi(\xi)$ as:

$\psi(\xi)=\frac{\mathrm{cK} \Gamma}{\xi}-\frac{\omega(\xi)}{\omega^{\prime}(\xi)} \phi_{*}(\xi)+\sum_{\mathrm{j}=1}^{2} \frac{\mathrm{h}_{\mathrm{j}} \phi_{*}\left(\mathrm{n}_{\mathrm{j}}^{-1}\right)}{1-\mathrm{n}_{\mathrm{j}} \xi}+\mathrm{B}(\xi)-\mathrm{B}$

where

$\phi_{*}(\xi)=\phi^{\prime}(\xi)+c \Gamma-\frac{X-i Y}{2 \pi(1-\chi) \xi}$,

$B(\xi)=\frac{1}{2 \pi i} \int_{\gamma_{j}}^{F-\xi} \frac{F(\sigma)}{\sigma-\xi} d \sigma$

and

$B=\frac{1}{2 \pi i} \int_{y_{j}} \frac{F(\sigma)}{\sigma} d \sigma$

\section{THERMOELASTIC POTENTIAL FUNCTION}

For two dimensional problems for thermoelastic infinite plate, we have $\sigma_{z x}=\sigma_{z y}=0$,

where $\sigma_{z x}$ and $\sigma_{z y}$ are the components of stress in the fixed direction $z$. Also from equilibrium equation:

$$
\begin{aligned}
& \frac{\partial \sigma_{x x}}{\partial x}+\frac{\partial \sigma_{y x}}{\partial y}=0 \\
& \frac{\partial \sigma_{x y}}{\partial x}+\frac{\partial \sigma_{y y}}{\partial y}=0
\end{aligned}
$$

From Fourier's law, $q$, the rate of heat flow per unit area is proportional to the temperature gradient in the direction of the normal $n$ to the element,

$q=-k \frac{\partial \theta}{\partial n}$

where $k$ is the thermal conductivity of the material, $\theta$ is the temperature difference and the minus sign is due to the fact that heat flows in the direction of decreasing temperature.

In the stationary case of thermoelastic infinite plate, we have the boundary value problem:

$\nabla^{2} \theta=0$

with the boundary condition: 
$\frac{b c}{\partial n}=0$ at $r=r_{0}$. (on the boundary) where $z=x+i y, x=r \cos \rho$ and $y=r \sin \rho$.

The solution of (4.3) from [14] is given by

$\theta=q\left(\operatorname{Im} z+\frac{r_{0}^{2} \sin ^{2} \rho}{\ln z}\right)$

At the origin $\theta=\mathrm{O}\left(\frac{1}{\mathrm{z}}\right)$, which has a greatest value as $\mathrm{z} \longrightarrow 0$,

Also when $\mathrm{z} \longrightarrow \infty$, the increasing temperature will take the value $\theta=q \mathrm{y}$.

Neglecting the variation of the strain and stress with respect to the thickness of the plate, so thermoelastic potential function $\Phi$ takes the form see Parkus (1976)

$\nabla^{2} \Phi=(1+v) \alpha 0$

Where $\alpha$ is the coefficient of thermal expansion and $v$ is the poisson's ratio.

In the case of uniform heat flow and by using (4.4) in (4.5), we get:

$(1)=\frac{(1+v) \alpha q r_{0}^{2}}{4}(\ln z+\ln z \bar{z})$

\section{STRESS COMPONENTS IN THE PRESENCE OF HEAT}

From Muskhelishvili and Parkus, the stress components satisfy the following equation:

$\sigma_{\mathrm{xx}}+\sigma_{y \mathrm{y}}=4 \mathrm{G}\left\lfloor\phi^{\prime}(\mathrm{z})+\overline{\phi^{\prime}(\mathrm{z})}-\lambda \theta\right]$

and

$\sigma_{x x}-\sigma_{y y}+2 i \sigma_{x y}=2 \mathrm{G}\left[\frac{\partial^{2}(1)}{\partial x^{2}}-\frac{\partial^{2}(D)}{\partial y^{2}}+2 i \frac{\partial^{2} \Phi}{\partial x \partial y}\right]-4 \mathrm{G}\left[z \overline{k^{\prime \prime}(z)}+\overline{\psi^{\prime}(z)}\right]$

Where $G$ is the shear modulus and $\lambda=\frac{1+v}{2} \alpha$ is the heat transfer coefficient.

The partial derivatives of $\Phi$ with respect to $x$ and $y$ takes the form:

$\frac{\partial^{2} \Phi}{\partial x^{2}}=\frac{(1+v) \alpha \sigma \mid l_{0}^{2}}{2} \frac{y^{3}-x^{2} y}{\left(x^{2}+y^{2}\right)^{2}}$

$\frac{\partial^{2} \Phi}{\partial y^{2}}=\frac{(1+u) \alpha y r_{0}^{2}}{2} \frac{3 x^{2} y+y^{3}}{\left(x^{2}+y^{2}\right)^{2}}$

$\frac{\partial^{2}(D)}{\partial x \partial y}=\frac{(1+u) \alpha q r_{0}^{2}}{2} \frac{x^{3}-x y^{2}}{\left(x^{2}+y^{2}\right)^{2}}$

Solving equations (5.1) and (5.2) together we get the stress components as $\left.\sigma_{x x}=-2 G\left\{\gamma_{i}^{*}-\operatorname{Re} \mid 2 \phi^{\prime}(z)-M(z, \bar{z})\right]\right\}$, 
$\sigma_{y y}=-2 G\left\{y_{2}^{*}-\operatorname{Re}\left[2 \phi^{\prime}(z)+M(z, \bar{z})\right]\right\}$, and

$\sigma_{x y}=G\left\{\gamma_{1}-4 G \operatorname{Im} M(z, \bar{z})\right\}$

where

$\gamma_{1}^{*}=\frac{\partial^{2} \Phi}{\partial y^{2}}, \quad \gamma_{2}^{*}=\frac{\partial^{2} \Phi}{\partial x^{2}}, \quad \gamma_{1}=\frac{\partial^{2} \Phi}{\partial y \partial x}$

and $M(z, \bar{z})=\overline{z \phi^{\prime \prime}(z)}+\overline{\psi^{\prime}(z)}$

So, the stress Components in the presence of heat are completely determined.

\section{REFERENCES}

Abdou, M.A. and Khar Eldin, E. A., 1994. An finite plate weakened by a hole having arbitrary shape, $d$. Comp. Appl. Math. 56: 341-351.

Abdou, M.A., and El-Bary, A. A., 2001. Fredholm-Volterra integral equation with potential kernel, Int. J. Math. and Math. Sci., 26(6): 321-330.

Abdou, M. A. and El-Bary, A. A., 2001. Fundamental problems for infinite plate with a curvilinear hole having finite poles, Math. Prob. In. Eng., 7(6): 485-501.

Aleksandrov, V. M. and Kovalenko, E. V., 1986. Problems with mixed boundary conditions in continuous mechanics, Nauka Moscow.

El-Bary, A. A., Ismail, I. S and Ewadh, S., 2003. Solution of First and second fundamental problems of an elastic infinite plate with Three poles, New Zeland. J. Math. 32(2): 1-9.

El-Bary, A.A. and El-Sirafy, I. H., 1999. An infinite plate with a curvilinear hole in s-plane, Estratto da le Matematiche vol. LIV- fasc II pp. 261-274.

El-Bary, A. A., 2000. Chebyshev polynomial solution for Volterra-Fredholmn integrodifferential equation with Cauchy kernel, Int.J.Appl. Math, 2(12): 1399-1406.

El-Bary, A. A. and Ismail, I. S., 2000. Solution of first and second fundamental problems of an elastic infinite plate, int. J. Appl. Math., 4(3): 247-260.

El-Bary, A.A., 2001. First and second fundamental problem of an elastic infinite plate with holes, Korean J. Comp. Appl. Math., 8(3): 675-684.

El-Sirafy, I. H., 1977. Stretched plates weakened by inner curvilinear holes, J. of Appl. Math and phys. (ZAMP), 28: 1153-1159.

England, A.H., 1971. Complex variable method in elasticity, London, New York.

Koya, A. C. and Erdogan, F., 1997. On the solution of integral equation with a generalized Cauchy Kemel, Quart. Appl. Math., X LV(3): $455-400$.

Muskhelishvili, N. I., 1949. Some basic problems of mathematical theory of elasticity, Moscow.

Parkus, H., 1976. Thermoelasticity, Springer Verlag, New York.

Popv, G. Ya., 1982. Contact problems for a linearly deformable function, Kiev, Odessa . 